\title{
Introduction to the Special Issue
}

Nanobioengineering is a rapidly growing field in biomedical engineering in which nano-scale science and engineering are being integrated and applied to studies in bioengineering, molecular biology and medicine. It focuses on the development of engineered nano-scale (1-300 nm) materials, structures, and devices for better understanding of biology and better diagnosis and treatment of diseases. In this Special Issue of Annals of Biomedical Engineering, we present a set of invited review articles and original technical reports that showcase the recent development and applications of nanoprobes, nanomaterials, nanocarriers, and nanoscale approaches in bioengineering, and demonstrate the potential of nanobioengineering in providing unprecedented opportunities in biomedical and biological studies.

The application of nanotechnology and nanobioengineering to biology and medicine is expected to produce significant breakthroughs in biological and biomedical research, as well as in medical diagnostics and therapeutics. In particular, multifunctional nanoparticles hold great promise for imaging and drug/gene delivery, thus providing a powerful tool for simultaneous disease diagnostics and therapeutics ("theranostics"). In this issue, Wickline provides a review of the application of perfluorocarbon nanoemulsions as molecular imaging agents and targeted drug delivery carriers in cancer and cardiovascular disease studies. This is followed by a set of papers by Mattoussi, Gao, Vazquez, and Beiler, respectively, to review the recent advances in developing quantum dot (QD) bioconjugates for biological studies, including encapsulation of single QDs with mesoporous silica, QD embedded microspheres for biosensing applications using Whispering Gallery Mode, the development of targeted QDs for the labeling of glial progenitor cells, and the use of QDs in studying biomolecular interactions, tracking of protein receptors, and constructing resonance energy transfer probes. The advantages offered and limitations encountered by QD based fluorescence probes are also discussed.

There is increasing interest in developing nano-scale carriers for drug/gene/probe delivery. The three review articles in this issue by Bellamkonda, Murthy, and Ho, respectively, discuss the recent advances in the development of nanocarriers for targeted therapy, including selective targeting of liposomal nanocarriers to neutrophils and monocytes using peptides, the development of disulfide-crosslinked polyion micelles for delivery of protein therapeutics, and the use of parylene-encapsulated copolymeric membranes for localized and sustained drug delivery. Although each of these papers focuses on a specific delivery system, the common scheme is to engineer robust nanomaterial-based drug/gene/protein delivery vehicles with specific targeting, better efficacy and controlled release. In some applications, there is a need to delivery nanoparticles or proteins into cell nucleus, so identifying alternative methods in nuclear import is important. In this regard, Bao provides a technical report to demonstrate that Tat peptide is able to delivery nanoparticles of $>40 \mathrm{~nm}$ across nuclear membrane.

Over the last few years a significant effort has been made to develop nanobioengineering as a new field. More application areas have been explored and new design principles for engineering nanosystems may emerge. In this special issue, Ly and Webster review, respectively, the development of fluorine-doped carbon nanotube biosensors for biomedical assays and the potential application of nanomaterials to regenerating tissues and possibly organs. Leary describes the nanobioengineering design process in which sophisticated nanoparticle systems are designed and integrated for diagnosis and treatment of disease. Guo, on the other hand, discusses the structure and function of a nanomachine, the phi29 DNA packaging motor, and its potential applications in medicine.

Nanomaterials and nanodevices have the potential to enjoy a wide range of applications in biology and medicine. However, nanobioengineering is still in its infancy and there exit many engineering challenges. For example, it is necessary to optimize nanoparticle size, surface chemistry, ligand type, and density to realize high signal specificity, enhanced local contrast, and reduced toxicity in in vivo molecular imaging applications. For delivery applications, it is important to tailor the nanostructure of the delivery vehicle to realize maximum payload, controlled, and activatable release, and specific targeting. Biocompatibility, cytotoxicity and safety of engineered nanoprobes, nanocarriers and nanodevices also present great challenges for in vivo applications. Although it may take years or even decades to translate nanobioengineering from bench to bedside in biomedical applications, with this special issue, we hope to stimulate a broader interest to develop and optimize multifunctional, self-assembled nanosystems that have unique properties unmatched by any existing engineered entities.

Gang BaO And Sohi Rastegar 